\title{
1 Targeted memory reactivation in human REM sleep 2 elicits recurrent, temporally compressed 3 reactivation
}

4

5

Mahmoud E. A. Abdellahi' ${ }^{1 *}$, Anne C. M. Koopman', Matthias S. Treder ${ }^{2}$ \& Penelope A. Lewis ${ }^{1}$

\begin{abstract}
Memories are reactivated during non-rapid eye movement (NREM) sleep, but the question of whether equivalent reactivation also occurs in rapid eye movement (REM) sleep is hotly debated. To examine this, we used a technique called targeted memory reactivation (TMR) in which sounds are paired with learned material in wake, and then re-presented in subsequent sleep to trigger reactivation. We then used machine learning classifiers to identify TMR-induced reactivation in REM. The reactivation we measured was temporally compressed by approximately five times during REM compared to wakeful performance of the task, and often occurred twice after a single TMR cue. Reactivation strength positively predicted overnight performance improvement and was only apparent in trials with high theta activity. These findings provide strong evidence for memory reactivation in human REM sleep after TMR as well as an initial characterisation of this reactivation.
\end{abstract}

\footnotetext{
${ }^{1}$ School of Psychology, Cardiff University Brain Research Imaging Centre (CUBRIC), Cardiff CF24 4HQ, United Kingdom.

${ }^{2}$ School of Computer Science and Informatics, Cardiff University, Cardiff CF24 3AA, United Kingdom. Correspondence and requests for materials should be addressed to: M.E.A.A. (email: abdellahime@cardiff.ac.uk) or A.C.M.K (email: koopman.anne@gmail.com) or P.A.L. (email: LewisP8@cardiff.ac.uk)
} 


\section{Introduction}

The reactivation of memories in non-REM sleep is widely supported by evidence from humans, rodents, and other animals ${ }^{1-4}$, but it is still unclear whether equivalent reactivation occurs in REM sleep. Reactivation in non-REM sleep has been identified using EEG classifiers ${ }^{3,5-7}$, with $\mathrm{fMRI}^{8,9}$, and with intracranial recording ${ }^{10}$. Targeted memory reactivation (TMR), a technique which allows the active triggering of memory reactivation, causes both neural and behavioural plasticity when applied in nonREM sleep, see ${ }^{11}$ for a review. However, only a few studies in rodents show evidence for reactivation in REM sleep ${ }^{12,13}$. Furthermore TMR in REM often fails to produce any measurable behavioural impact ${ }^{9,14}$, though conditioning seems to be the exception to this rule $e^{15,16}$. One prior study elegantly demonstrated that machine learning applied to EEG can be used to distinguish between the REM sleep occurring after training on two different tasks ${ }^{17}$. The authors also found a correlation between the extent to which such patterns were identified in NREM and subsequent behavioural performance, however they did not look for reactivation in terms of wake-like task related activity that reoccurs during REM. In the current study we do just this, and are thus able to establish a direct link between brain activity associated with a memory in wake and the reactivation after a TMR cue in REM. We also explore the temporal characteristics and dynamics of this reactivation.

Memories have a different temporal structure during reactivation compared to encoding. For instance, replay during non-REM sleep has been shown to occur 10 to 20 times faster than in wake ${ }^{4,18}$. On the other hand, wakeful and NREM1 reactivation in humans has also been demonstrated to be both compressed and dilated within the same participant ${ }^{19}$. Given this literature, we looked for evidence of temporal compression or dilation during memory reactivation in human REM sleep. Memory reactivation can reoccur more than once after a TMR cue in non-REM sleep ${ }^{6}$, and this may be of functional significance for consolidation from the hippocampus to the cortex ${ }^{11}$. We therefore sought to 
50 determine whether a similar pattern is apparent for REM reactivation. Additionally, theta activity is

51 prominent in REM sleep ${ }^{20}$, and human studies suggest a possible relationship between memory and

52 theta ${ }^{21,22}$. Wakeful theta is suggested to provide a preferable window for the encoding of new

53 information ${ }^{23-25}$. Of the few rodent studies that have supported memory reactivation in REM, two

54 showed a link to theta activity ${ }^{12,26}$. We were therefore interested to determine whether theta is

55 associated with TMR cued reactivation in human REM sleep.

56 We used a serial reaction time task (SRTT), which is known to be sleep sensitive ${ }^{27,28}$ to examine these

57 questions. Participants were presented with audio-visual cues and responded by pressing four buttons

58 (two from each hand). Cues were organised in a twelve-item sequence. Sounds were replayed during

59 subsequent REM to trigger the associated memories of left- and right-hand presses (Fig. 1). We used

60 two sequences and replayed only one of them in sleep. For control, we also included an adaptation

61 night in which participants slept in the lab and we played the same tones that would later be played

62 during the experimental night. This provided data in which tones could not have evoked memory

63 reactivation, as participants had not yet learned the behavioural task, see methods for more details

64 about the SRTT.

65 In summary, we discriminate between neural reactivation of left- and right-hand button presses using

66 linear classification of time domain EEG amplitudes during REM sleep. Our findings demonstrate that

67 such classifiers can detect reactivation after TMR cues in REM sleep, and that classification strength

68 predicts task overnight improvement. We also reveal several key characteristics of this TMR cued

69 reactivation, including recurrence, temporal compression, and an association with high theta activity. 


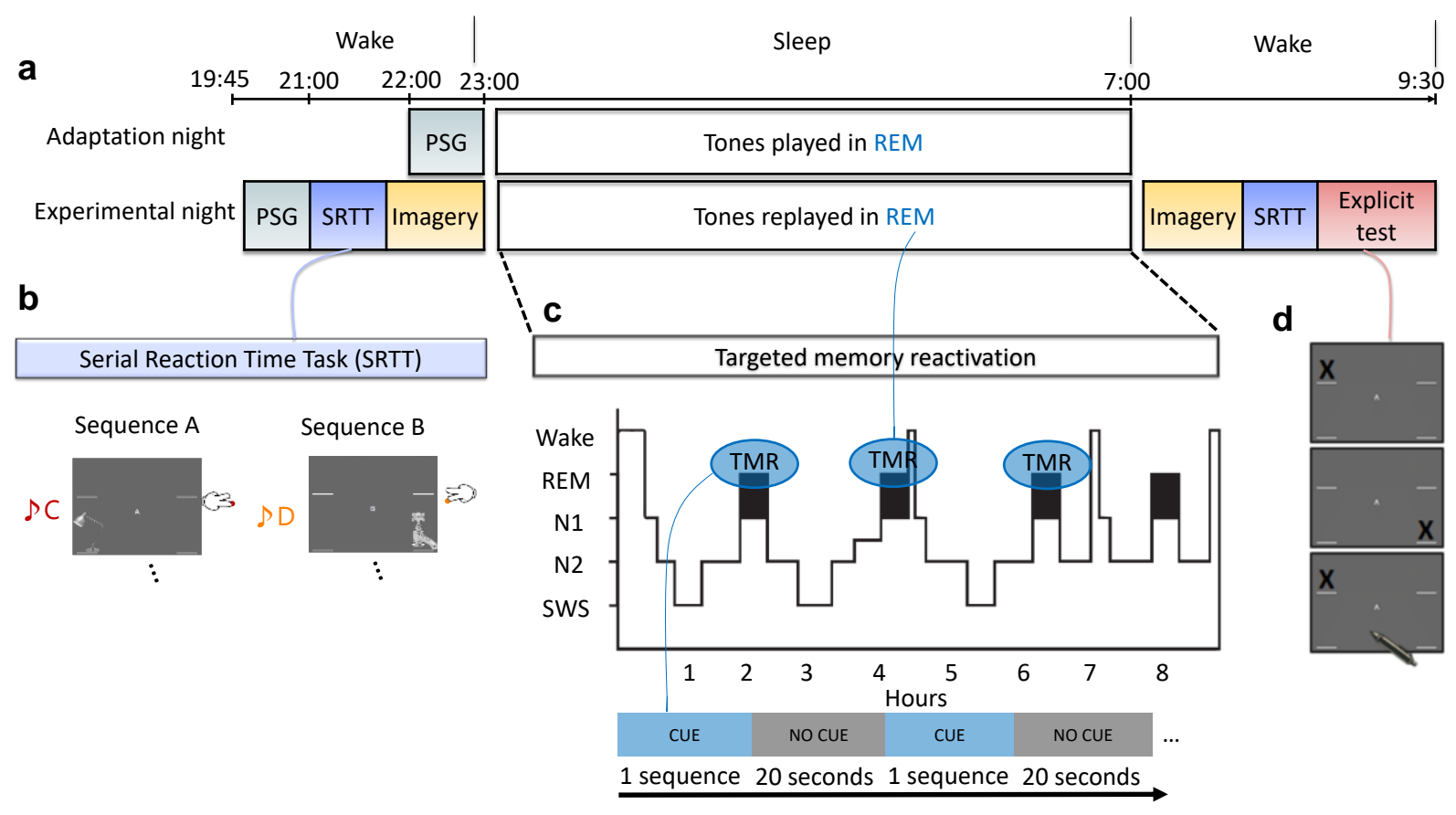

Fig. 1 Experimental design. a The experiment consisted of two nights an adaptation and an

73 experimental night. In the adaptation night, tones were presented to the participants during REM sleep and EEG recordings were acquired. In the experimental night, participants were wired-up for EEG then completed the serial reaction time task (SRTT) and an imagery task in which they were cued with pictures and sounds, but only imagine performing the finger tapping (without movement). Afterwards participants slept in the lab and TMR cues were presented. After waking up, participants completed the motor imagery and SRTT again, and finally they did the explicit recall task. $\mathbf{b}$ In the SRTT, images are presented in two different sequences each with a different set of tones. Each image is associated with a unique tone and requires a specific button press. In the imagery task, participants hear the tones and

81 see the images as in the SRTT, but only imagine pressing the buttons. c The sounds of only one learned 82 sequence (cued sequence) were played during REM sleep, with a 20 second pause between repetitions. remember during the explicit recall test after sleep. 


\section{Results}

\section{Detection of memory reactivation after TMR cues}

87 We trained our classification models using sleep data and then tested them on wake data. This was

88 partly inspired by Loui and Wilson ${ }^{12}$ who took a template from sleep data and slid it across wake to detect replay in rodents. Training a classification model on wake could cause the model to weigh features which are dominant in wake very highly even if those features were entirely absent from sleep. By training classification models on sleep data, we ensured that the features associated with reactivation were used by the models, and the models were thus able to look for these in the stronger, less noisy, signals recorded during wake.

We used a linear discriminant analysis (LDA) classifier in a time $\mathrm{x}$ time classification procedure ${ }^{29}$, see methods for details. We repeated the classification process using the adaptation night to be certain that the classification was not caused by sound induced effect or EEG noise rather than reactivation of the

97 encoded memory. We compared the results from the two nights, both to each other and to chance level using cluster-based permutation. In the adaptation night, no significant classification strength was found compared to chance (area under the receiver operating characteristic curve $(A \cup C)=0.5$ ), demonstrating that classification of this control condition did not differ from chance level. By contrast, comparison of the experimental night against chance showed a significantly higher AUC for the experimental night (Fig. experimental night to the adaptation night also showed a significantly higher AUC for the experimental night, described by a cluster in the same timeframe (Fig. 2a). This means that we can detect memory reactivation and discriminate between right- and left-hand movements during REM sleep, and this is significant when evaluated against both control and chance level. 


\section{High theta activity mediates reactivation}

109

110

111

112

113

114

115

116

117

118

119

120

121

122

123

124

125

126

127

128

129

To test for a relationship between theta power and reactivation we performed a median split on theta power, creating two groups of trials for each participant: those with high theta power and those with low theta power, see methods. This split was performed for both experimental and adaptation nights. We then compared the classification results of each half of the median split (high and low theta power trials) in the experimental night to both chance level and to the equivalent high or low theta power trials in the adaptation night. For high theta trials, this showed a significant effect (Fig. 2c), explained by a cluster occurring around the same time as in the classification result using all trials, (Fig. 2a-b), $(n=14, p$ $=0.028)$, there was also a significant difference against chance level (Fig. $2 d),(n=14, p=0.001)$. The low theta power trials showed no significant effect (Supplementary Fig. 3). These findings demonstrate an association between high theta power and reactivation. To determine whether theta activity offered a preferred window for classification, or it was the actual feature causing the discrimination of classes, we band-pass filtered the signals in the theta band and re-ran our classification analysis. Interestingly, classification did not differ from chance in this filtered data $(p>0.4)$ suggesting that while high theta activity offers a preferred window for reactivation, theta activity itself does not carry the information used to detect reactivation. Time $x$ time classification AUC plots using all trials and also using high theta power trials are shown in (Supplementary Fig. 2). 
Experimental vs. control

a

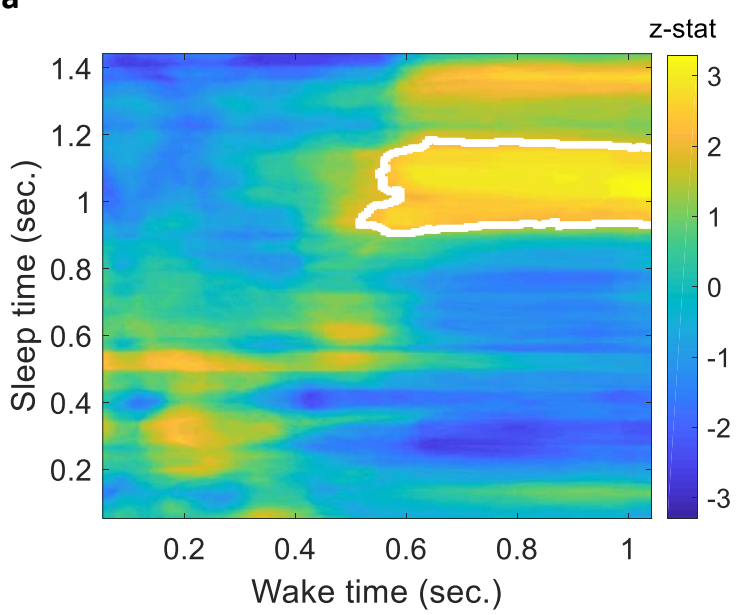

Experimental vs. chance

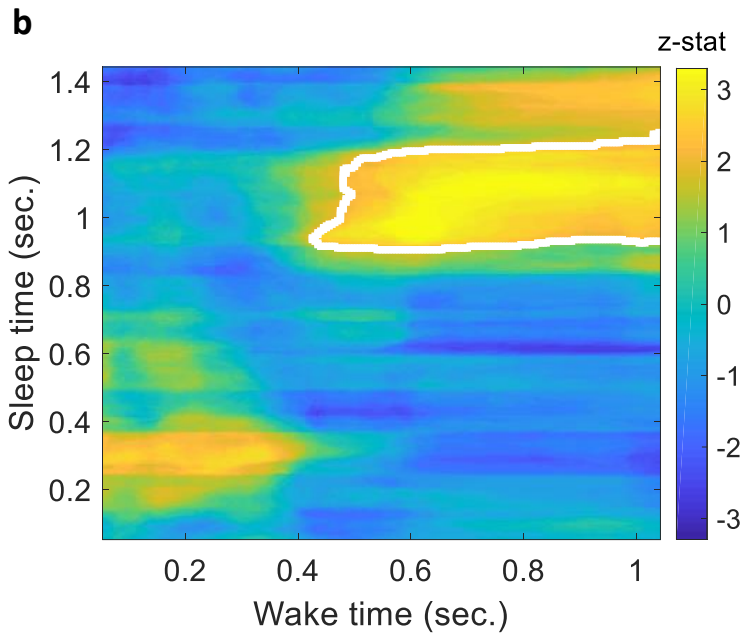

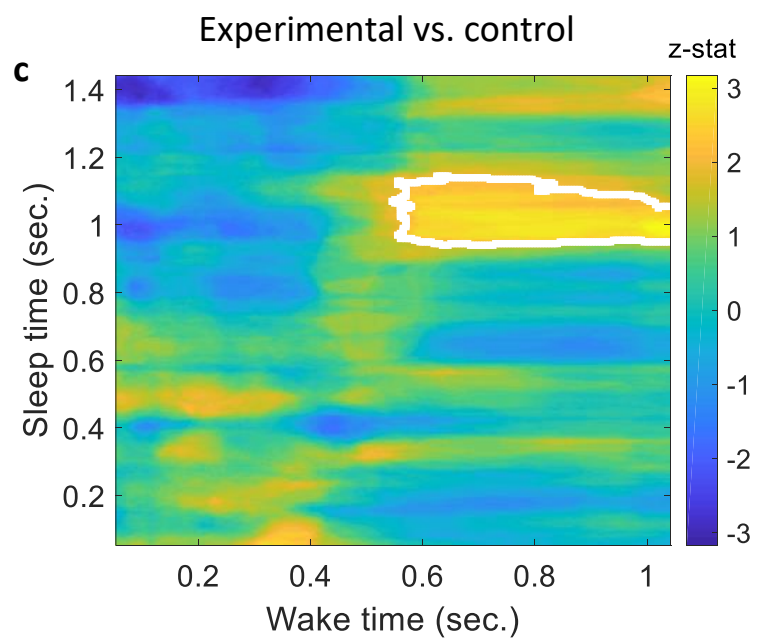

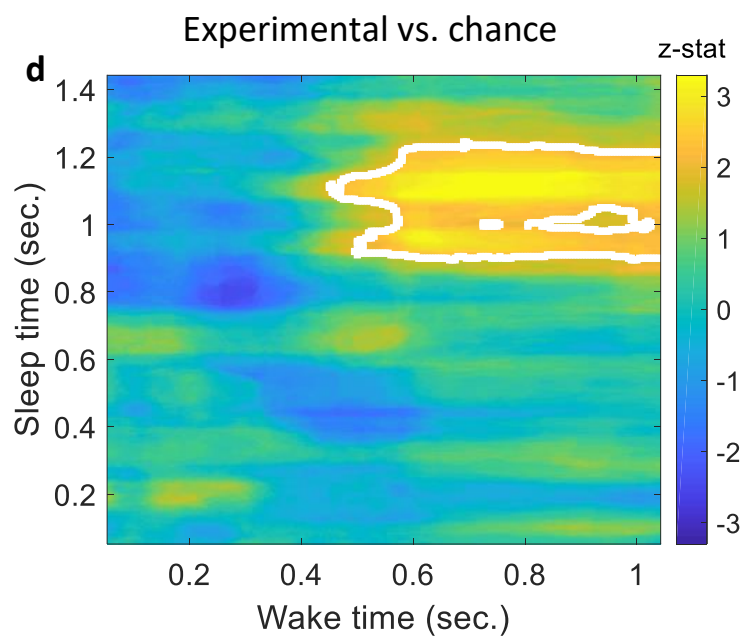

Fig. 2 Classification of left- vs. right-hand. a A comparison between the classification performance of the experimental vs. adaptation night using all trials reveals a significant effect described by a cluster which shows a higher classification performance for the experimental night compared to adaptation $(\mathrm{n}=$ $14, p=0.01)$, z-statistics values at every point is shown and cluster edges are marked with white after correcting for multiple comparisons with cluster-based permutation (see methods for details). b 
$1420.5)$ as shown by the corrected cluster $(n=14, p<0.0001)$. c A comparison between the experimental

143 and adaptation night classification using trials with high theta power reveals a significant effect

144 described by a cluster that shows a higher classification performance for the experimental night

145 compared to adaptation $(n=14, p=0.028)$, $z$-statistic values at every point is shown and corrected

146 cluster edges are marked with white. $\mathbf{d}$ A comparison between the classification of experimental night

147 using trials with high theta power and chance level shows a significant effect described by the shown

148 corrected cluster $(n=14, p=0.001)$.

\section{Correlation of classification performance with behaviour}

151 We next tested for a relationship between classification performance and improvement on the

152 behavioural task using a spearman correlation, see methods for details. This revealed a positive

153 correlation (Fig. 3a; $n=14, r=0.67$, uncorrected $p=0.01$ and Bonferroni corrected $p=0.03$ ). indicating

154 that stronger detected reactivation was associated with greater overnight sequence improvement.

155 Importantly, this correlation only existed for the reactivated sequence (for non-reactivated: $n=14, r=$

$1560.02, p=0.94)$. This finding suggests that the degree of detecting reactivation in REM positively predicts

157 the extent to which sequence memory improves overnight. Nevertheless, it is notable that REM TMR did

158 not lead to any overall benefit in performance ${ }^{30}$.

\section{Analysis of temporal compression/dilation of reactivation}

Prior work has shown that reactivation in non-REM sleep is often temporally compressed with respect to

161 wake ${ }^{4,18,31}$. Recent finding showed that wake and NREM1 reactivation could be sometimes compressed

162 and sometimes dilated within the same data ${ }^{19}$. This motivated us to determine whether reactivation in

163 REM occurs for the same amount of time as the original experience in wake. Thus, we performed an 
analysis of temporal compression. First, we applied the time $x$ time classification method on EEG

171 This suggests that reactivation of the memory in REM sleep is approximately five times faster than the

172 activation in wake when the memory was encoded.

a

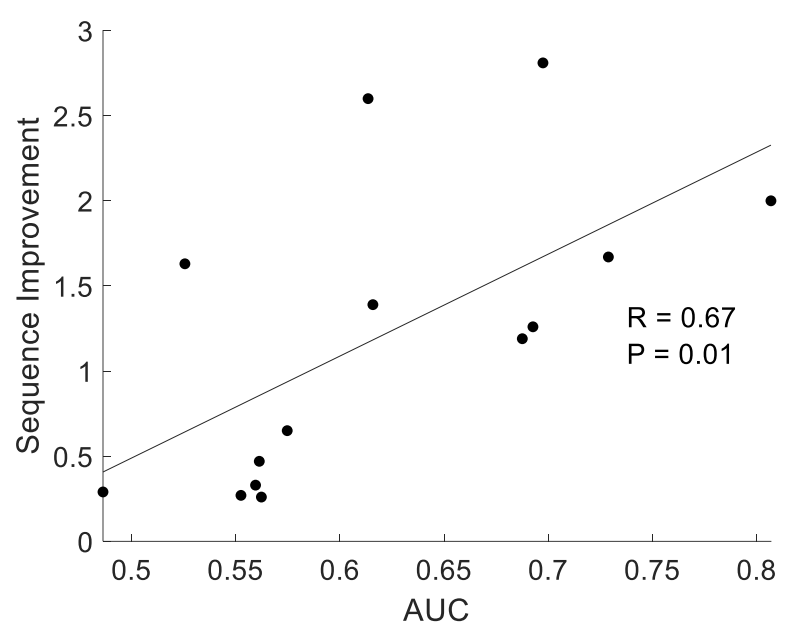

b

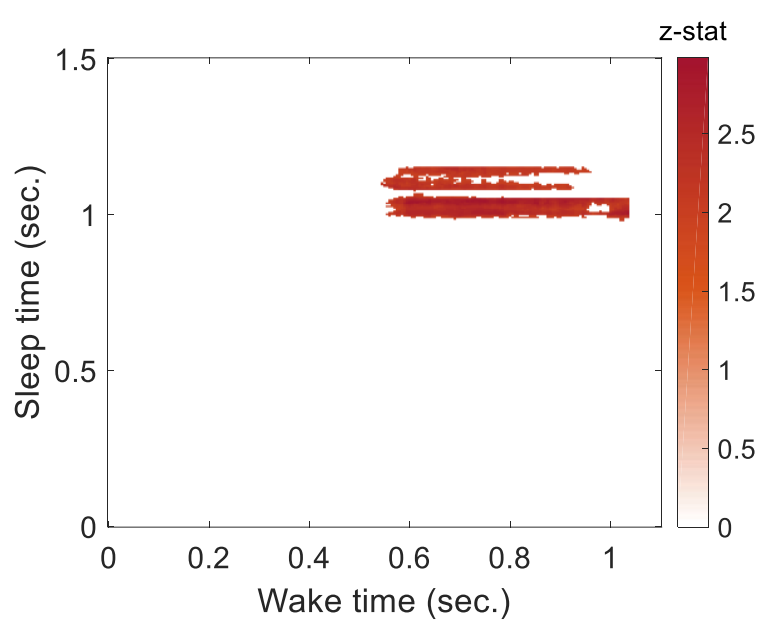

Fig. 3 Characteristics of detected reactivation. a Classification performance positively correlated with behavioural improvement for the reactivated sequence (spearman correlation, $n=14, r=0.67$,

177 uncorrected $p=0.01$ and Bonferroni corrected $p=0.03)$. $b$ Classification performance using EEG signals

178 without smoothing showed two significant effects described by two clusters, around $1 \mathrm{sec}$. after the 179 onset of the cue when compared to the adaptation night ( $n=14, p=0.008$ for the earlier and, 0.025 for the later cluster). Z-statistic values are shown and cut with respect to clusters to become visually clearer. 
181 Locations of significant effects and their temporal characteristics reveal that reactivations are

182 approximately five times faster than the activation in wake.

\section{Reoccurrences of reactivation}

Finally, we were interested to know if TMR cued reactivation in REM occurs multiple times within each

trial, or if the apparent recurrence might instead be due to evaluating performance across multiple trials, with reactivation only happening once during each trial (e.g. at $\sim 1 \mathrm{sec}$. or after $1 \mathrm{sec}$, but not both).

To address this, we evaluated the correctness of each sleep trial at the times of each of the two subclusters, see methods for details. We then determined whether classification was correct for both sub-

190 clusters or just one sub-cluster for every trial (Fig. 3b). We found that a significantly higher proportion of

191 trials show reactivation at both sub-clusters (early and late), than at just one (Wilcoxon signed-rank test,

$192 \mathrm{n}=14, \mathrm{p}=0.001$ ). This shows that reactivation is recurrent in the majority of trials, and could potentially

193 repeat even more than twice if the duration of trials was longer. This is in keeping with observations

194 from rodent data showing repeated reactivation after a TMR cue in non-REM sleep².

\section{Discussion}

196 We have demonstrated that memory reactivation can be detected in human REM sleep using EEG

197 classifiers. Such reactivation appears to be delayed by about one second after the sound cue onset.

198 Reactivation is associated with high theta power, which appears to provide a preferred window for this

199 activity, although it does not carry the discriminative information needed to actually detect the

200 reactivation. Interestingly, reactivations reoccur twice within a single 1,500 ms trial and are temporally

201 compressed approximately five times compared to wake. We also demonstrated that stronger detection 
of REM reactivation predicts greater overnight sequence improvement on our behavioural task, and this

203 is specific to the reactivated sequence.

Because we used a motor task, we used motor channels for classification. This ensured that the

reactivation we measure is related to the encoded motor memory and that detected reactivation retains

208 Reactivation after a reminder TMR cue is delayed during REM sleep as compared to wake. During wake, neural activity associated with our motor imagery task starts around 0.6 seconds after cue onset and lasts until the presentation of the next cue at 1.1 seconds post cue. In REM, on the other hand, reactivation of

211 this task starts around 1 second after the cue and ends at around 1.25 seconds post cue. This delayed

212 start could potentially be due to the brain taking more time to process information and reactivate a 213 memory during REM sleep than during wake. In keeping with this, reactivation of a picture memory task

214 in SWS has also been shown to be delayed, appearing about 2 seconds after cue onset ${ }^{7}$.

215 Our results show that classification performance in REM positively predicts overnight sequence

216 improvement. Similar correlations between classification performance and behaviour were found in

217 non-REM TMR ${ }^{3,8,10}$ and also spontaneous reactivation ${ }^{17}$. These findings could lead to the speculation that

218 more reactivation means more consolidation, and therefore better post-sleep performance. However

219 the absence of a group-level REM TMR benefit in our behavioural performance (see ${ }^{30}$ for a full analysis

220 of the behavioural data), as well as the fact that other studies have observed negative correlations

221 between reactivation evidence and post-sleep improvement ${ }^{32}$ mean we must treat such interpretations

222 with caution.

223 REM sleep is dominated by theta activity, which is thought to support the consolidation process ${ }^{33}$, and

224 has been linked to reactivation ${ }^{12}$. Theta is linked to attention during wake ${ }^{34}$ and is more prominent with 
225 higher executive control ${ }^{35}$. Wakeful theta is also associated with the encoding of new information and 226 memory processing ${ }^{25}$. Furthermore, neuronal firing relative to theta phase has been shown to impact 227 upon whether synapses are strengthened or weakened, since stimulation of the positive theta phase 228 induces long-term potentiation and stimulation of the negative theta phase induces depotentiation ${ }^{36}$. A 229 similar pattern of phase dependent potentiation and depotentiation was shown in REM sleep ${ }^{26}$, 230 suggesting that REM reactivation and wakeful pattern may be structured in a similar way ${ }^{23,37}$. Theta 231 phase similarity has been shown to be high between SWS and wake, suggesting the importance of theta 232 phase for reactivation ${ }^{6}$. Increased theta power has also been shown to be important for successful cueing during sleep ${ }^{38}$. Our data demonstrated that trials with higher theta power show greater evidence

234 of memory reactivation, but theta band in isolation does not provide sufficient information to detect

235 reactivation. Theta therefore appears to be a marker of reactivation but does not embody the 236 reactivation in and of itself. This pattern of results could indicate that theta provides a timing function 237 for reactivation, while the reactivation itself relies upon other frequencies.

238 Studies in rats have shown that replay is temporally compressed with respect to 'run', or performing the 239 task in question, and the rate of that compression varies between sleep and wake. For instance, replay 240 in both wake and SWS has been shown to occur at a faster rate than in the original task ${ }^{18,39,40}$. However 241 some studies of rodent non-REM sleep showed a compression of 6 to 7 times compared to wake ${ }^{31}$, while 242 other studies showed compression rates varying between 10 and 20 times compared to wake $e^{4,18}$. Our 243 analysis is in line with this literature, since it suggests that TMR reactivation in human REM sleep is 244 temporally compressed by approximately 5 times compared to wake.

245 The reactivation elicited by TMR in human SWS has been shown to reoccur repeatedly after a cue ${ }^{6}$. A 246 parallel study in rodents ${ }^{2}$ showed that TMR cued replay can continue to repeat for up to 10 seconds 247 after the offset of the auditory cue, though this process appears to be interrupted by the presentation of 248 a second stimulus. These observations of repeated replay have been suggested to relate to the 

reverberation of replay between the cortex and hippocampus ${ }^{41}$, although one might potentially expect such reverberation to occur on a shorter timescale. We tackled the question of whether replay occurs repeatedly after a single TMR cue in REM and found two repetitions following a single TMR cue, which is in good keeping with the results from previous studies ${ }^{2,6}$. Notably, our trials were just 1.5 seconds long, so it is possible that further reoccurrence would have been observed in our paradigm if the cues were spaced farther apart.

\section{Characteristics of reactivations that happen in SWS, and REM sleep}

257 While there is already a growing body of literature about reactivation in non-REM sleep ${ }^{1-4,7,12,18,42}$, our

258 findings provide initial information about human memory reactivation using TMR in REM. As such, our 259 data suggests several parallels between reactivation in these two sleep stages. For instance, similar to 260 non- $\mathrm{REM}^{7}$, reactivation in REM is delayed after cue onset compared to wake. Furthermore, reactivation

261 in REM is related to the oscillatory structure of sleep (e.g. theta activity), which parallels the known 262 relationship between reactivation in non-REM and graphoelements like $\mathrm{SOs}^{43}$, spindles ${ }^{7,44}$, and ripples $^{10}$.

263 Importantly, the reactivations we detected are reinstated in the same area of the brain that is used in

264 performing the task and the extracted features of reactivation are similar to those extracted during

265 wake activation which is why it is detectable using our machine learning model. Finally, REM

266 reactivations appear to be temporally compressed by about five times in comparison to wake, which is 267 close to the 6 to 7 times compression observed in non-REM ${ }^{31}$.

268 Summary

The question of whether memories reactivate in REM as well as non-REM sleep has been debated for some years. REM reactivation has been suggested by modelling ${ }^{45}$ and has been demonstrated at least

271 once in the rodent literature ${ }^{12}$. Some evidence of learning dependent activation in human REM sleep has 
272 also been observed in humans ${ }^{46,47}$. However, null behavioural findings from human REM TMR studies ${ }^{1,9}$

273 have led to scepticism amongst sleep researchers. Our current findings put such doubts to bed by

274 providing clear evidence that TMR in REM is associated with reactivation, and even predicts overnight

275 improvement on our procedural task, although we found no significant benefit from cueing irrespective

276 of reactivation. Furthermore, our analysis uncovers several important properties of REM reactivation,

277 showing strong parallels with reactivation in non-REM sleep. More work is needed to explore this topic

278 in detail, for instance determining how such reactivation links to behavioural and neural plasticity, and

279 how this differs across a variety of cognitive tasks.

\section{Methods}

\section{Participants}

282 EEG and behavioural data were collected from human participants $(n=16)(8$ females, 8 males, and age mean: 23.6). All participants had normal or corrected-to-normal vision, normal hearing, and no history

284 of physical, psychological, neurological, or sleep disorders. Responses in a pre-screening questionnaire 285 reported no stressful events and no travel before commencing the study. All participants reported non286 familiarity with the SRTT and all of them were right-handed. Participants did not consume alcohol in the

28724 hours before the study and caffeine in the 12 hours prior to the study or perform any extreme

288 physical exercise or nap. This study was approved by the School of Psychology, Cardiff University

289 Research Ethics Committee, and all participants gave written informed consents.

\section{Experimental Design}

291 The SRTT was adapted from ${ }^{48}$ and participants performed SRTT before and after sleep. Two participants 292 were excluded due to technical problems $(n=14)$. In SRTT, sounds cued four different finger presses. 
and B: 24323142314 1. The location indicated which key on the keyboard to press as quickly and

295 accurately as possible afterwards the next image appeared, locations and buttons associations were: 1 -

296 top left corner = left shift key; 2 - bottom left corner = left Ctrl; 3 - top right corner = up arrow; 4 -

297 bottom right corner = down arrow. Sequences had been matched for learning difficulty; both contained

298 each item three times. The blocks were interleaved so that a block of the same sequence was presented

299 no more than twice in a row, and each block contained three repetitions of a sequence. There were 24

blocks of each sequence (48 blocks in total), after each block a 15-second pause which could be

extended by participants if they wish, during the pause participants were informed with their reaction

time and error rate for the last block. The 48 blocks of sequences $A$ and $B$ were followed by four blocks

314 for each sequence ( $1=$ male face, $2=$ lamp, $3=$ female face, $4=$ water tap $)$. Visual cues stayed on the

315 screen until the correct key was pressed, after which an 880 ms inter-trial interval followed. After

316 completing the SRTT, participants performed the imagery task by only imagining pressing the buttons,

317 without movement. This task consisted of 30 interleaved blocks (15 of each sequence) and was 
presented in the same order as during the SRTT. Each trial consisted of a tone and a visual stimulus, the latter being shown for $270 \mathrm{~ms}$ and followed by an $880 \mathrm{~ms}$ inter-trial interval. There were no random blocks during the imagery task and no performance feedback during the pause between blocks. As a

321 control, participants were asked to sleep in the lab before doing the SRTT training. The control night

322 followed the same criteria as the actual experiment with the difference that the sounds were not

323 associated with any task. Participants performed the task again in the morning but this time motor

324 imagery then SRTT. They were then asked to try to remember the locations of the images of the two 325 sequences to test their recall for each of the sequences.

\section{EEG recording and pre-processing}

327 EEG signals were acquired from 21 electrodes, following the 10-20 system. Thirteen electrodes were 328 placed on standard locations: FZ, CZ, PZ, F3, F4, C5, CP3, C6, CP4, P7, P8, O1, and O2. Other electrodes

329 were: left and right EOG, three EMG electrodes on the chin, and the ground electrode was placed on the 330 forehead. Electrodes were referenced to the mean of the left and right mastoid electrodes. The 331 impedance was $<5 \mathrm{k} \Omega$ for each scalp electrode, and $<10 \mathrm{k} \Omega$ for each face electrode. Recordings were 332 made with an Embla N7000 amplifier and RemLogic 1.1 PSG Software (Natus Medical Incorporated). PSG 333 recordings were scored by trained sleep scorers and only the segments scored as REM were kept for 334 further analyses.

335 Data were collected at $200 \mathrm{~Hz}$ sampling rate. EEG signals were band pass filtered in the frequency range

336 from ( 0.1 to $50 \mathrm{HZ}$ ). Subsequently, trials were cleaned based on statistical measures consisting of 337 variance and mean. Trials were segmented $-0.5 \mathrm{sec}$. to $3 \mathrm{sec}$. relative to the onset of the cue. Trials 338 falling two standard deviations higher than the mean were considered outliers and rejected if they show 339 to be outliers for more than $25 \%$ of the channels. If trials were bad in less than $25 \%$ of the channels, they were interpolated using triangulation of neighbouring channels. Thus, $9.8 \%$ and $10.5 \%$ of trials 
341 were considered outliers and removed from the experimental night data and the adaptation night,

342 respectively.

343 Data was subsequently analysed with independent component analysis (ICA), to remove eye movement

344 artifacts which can occur during REM. Components identified by the ICA that were significantly

345 correlated with the signals from the eye electrodes (corrected for multiple comparisons) were removed.

346 All channels for each participant were manually inspected and artifacts were removed. Because TMR will

347 not be effective with all trials, we also rejected trials with low variance that do not differ from their

348 mean across time since they are unlikely to contain a response. The number of clean trials kept after

349 cleaning was consistent among participants such that they contribute equally to the group-level analysis

350 and that number was 366 trials, it was determined according to the participant with the lowest number

351 of such trials. All cleaning was done on all trials irrespective of cue information and stimulation night to

352 avoid bias.

\section{Time $\mathbf{x}$ time classification with time domain features}

354 We adopted a time $\mathrm{x}$ time classification approach after smoothing the EEG signals of motor imagery using 100 ms window such that every time point is replaced with the average of the 50 ms of both sides around it. Since we know that this task is motor-dependent we focused our classification on the motor

357 area, thus we used four channels around the motor area for classification (C5, CP3, C6, and CP4). In the

358 time $\mathrm{x}$ time classification, every time point from sleep was used to train LDA classifier, which was applied

359 to all time points from wake in order to get one row of classification results in the time $x$ time

360 classification plot (e.g., Fig. 2a). The process was repeated for all time points after a sound cue in sleep

361 (trial length in sleep was: $1.5 \mathrm{sec}$. and $1.1 \mathrm{sec}$. in wake) (Supplementary Fig. 1). We use the area under

362 the receiver operating characteristic curve (AUC) as the performance measure in our classification.

363 Analyses were done using FieldTrip ${ }^{49}$ and customised scripts using Matlab 2018a. The clustering window 
used for cluster-based permutation was the whole length of trials (i.e., the whole time $x$ time

365 classification duration) which gave a stringent test. In other words, we did not limit the clustering

366 window to a specific time window.

\section{Theta power calculation}

368 We calculated theta power using band pass filtering in the range ( 4 to $8 \mathrm{~Hz}$ ) and Hilbert transform to 369 extract the instantaneous power information of the signals. The power of a trial is calculated as the

370 average power across all time points of that trial and all channels. We then divided the trials of every

371 participant into two groups based on the median power of trials. This gave us trials with high theta

372 power (higher than median) and low theta power (lower than median).

\section{Temporal compression of reactivation}

374 We analysed the temporal compression by applying the time $x$ time classification using the EEG

375 amplitudes without smoothing the EEG signals. We used the cluster we found from the first

376 classification (around $1 \mathrm{sec}$.) as a clustering window, we used this window as a marker of time of interest

377 because if the reactivation is compressed then it will be temporally short. Duration of sleep reactivation

378 is calculated as the average durations of the two sub-clusters in sleep time. Duration of wake activation

379 was determined by the average durations of the sub-clusters in wake time (Fig. 3b).

380 Correlation of classification performance with behaviour

381 Classification performance was averaged inside the cluster. AUC values from the high theta power

382 classification were tested for correlation with the behavioural improvement. The behavioural

383 improvement was calculated as: [(random blocks after sleep - the best 4 blocks after sleep) - (random

384 blocks before sleep - the best 4 blocks before sleep)] / (random blocks before sleep - the best 4 blocks

385 before sleep). The result was corrected for other measures using Bonferroni correction. We extracted 
three behavioural measures: early blocks improvement, late blocks improvement, best blocks

improvement (described above). Early blocks improvement was defined as: [(random blocks after sleep the first 4 blocks after sleep) - (random blocks before sleep - the last 4 blocks before sleep)] / (random blocks before sleep - the last 4 blocks before sleep). Late blocks improvement was defined as: [(random blocks after sleep - the last 4 blocks after sleep) - (random blocks before sleep - the last 4 blocks before sleep)] / (random blocks before sleep - the last 4 blocks before sleep).

\section{Reoccurrence}

We analysed whether the classification is reoccurring within the same trial or whether it happens once while it only appears to be reoccurring as a result of looking at the performance among multiple trials. For this, we under-sampled wake trials, that is, we used wake time according to the two sub-clusters in

(Fig. 3b) and under-sampled the wake trial to match the length of sleep cluster. Then, for every trial of sleep, we performed spearman correlation with all trials from wake such that we ended with one vector of correlation coefficients for every trial from sleep. Subsequently, we counted the number of times a trial from sleep positively correlated with wake trials of the same class, and the other class. A trial from

400 sleep is then considered 'correct' if the number of positive significant correlations is higher for similar 401 class than different class. Afterwards, we determined if reoccurrence is happening in sleep trial if it is 402 correct during both the earlier and later clusters. Then we counted the number of trials with different 403 types: recurrent, only earlier cluster, and later cluster. Afterwards, we normalised by the total number 404 of trials for every participant which gave a datapoint for every participant for every type. Subsequently, 405 the number of trials for every type was compared to chance 0.333 and recurrent reactivation was the 406 only type that showed higher than chance trial count (Wilcoxon signed-rank test, $n=14, p=0.001$ ). On

407 the other hand, earlier cluster only: (Wilcoxon signed-rank test, $n=14, p=0.0009$ ) was below chance. 


\section{Correcting for multiple comparisons}

410 Multiple comparisons correction was done using MVPA-Light toolbox in Matlab ${ }^{50}$ and customised

411 scripts. Cluster-based permutation testing was used with sample-specific threshold of 0.05 . Permutation

412 test threshold for clusters was 0.05 , and 10,000 permutations were calculated.

\section{Data availability}

415 All relevant data generated or analysed are available from the authors upon reasonable request,

416 including the EEG data, behavioural files, all the analyses with MATLAB scripts. Participants private

417 identifications are all anonymized.

\section{Acknowledgements}

419 This work was supported by the ERC grant SolutionSleep to P.A.L and ERC funded the Ph.D. of M.E.A.A.

420 We would like to thank Martyna Rakowska and Lorena Santamaria and all members of our group NaPs

421 for the useful comments.

\section{Author contributions}

423 A.C.M.K. and P.A.L. designed the experiment. A.C.M.K collected the data. M.E.A.A analysed the data.

424 M.E.A.A., P.A.L., A.C.M.K. and M.S.T wrote the manuscript.

425 Supplementary Information are available online.

426 Competing interests: The authors declare no competing interests. 


\section{References}

1. Rasch, B. \& Born, J. About sleep's role in memory. Physiol. Rev. 93, 681-766 (2013).

2. Bendor, D. \& Wilson, M. A. Biasing the content of hippocampal replay during sleep. Nat.Neurosci. 15, 1439-1444 (2012).

3. Wang, B. et al. Targeted Memory Reactivation during Sleep Elicits Neural Signals Related to Learning Content. J. Neurosci. 39, 6728-6736 (2019).

4. Ji, D. \& Wilson, M. A. Coordinated memory replay in the visual cortex and hippocampus during sleep. Nat. Neurosci. 10, 100-107 (2007).

5. Belal, S. et al. Identification of memory reactivation during sleep by EEG classification. Neuroimage 176, 203-214 (2018).

6. Schreiner, T., Doeller, C. F., Jensen, O., Rasch, B. \& Staudigl, T. Theta Phase-Coordinated Memory Reactivation Reoccurs in a Slow-Oscillatory Rhythm during NREM Sleep. Cell Rep. 25, 296-301 (2018).

7. Cairney, S. A., Guttesen, A. á. V., El Marj, N. \& Staresina, B. P. Memory Consolidation Is Linked to Spindle-Mediated Information Processing during Sleep. Curr. Biol. 28, 948954.e4 (2018).

8. Shanahan, L. K., Gjorgieva, E., Paller, K. A., Kahnt, T. \& Gottfried, J. A. Odor-evoked category reactivation in human ventromedial prefrontal cortex during sleep promotes memory consolidation. Elife 7, 1-21 (2018).

9. Rasch, B., Buchel, C., Gais, S. \& Born, J. Odor Cues During Slow-Wave Sleep Prompt Declarative Memory Consolidation. Science (80-. ). 315, 1426-1429 (2007).

10. Zhang, H., Fell, J. \& Axmacher, N. Electrophysiological mechanisms of human memory consolidation. Nat. Commun. 9, 4103 (2018).

11. Lewis, P. A. \& Bendor, D. How Targeted Memory Reactivation Promotes the Selective Strengthening of Memories in Sleep. Curr. Biol. 29, R906-R912 (2019).

12. Louie, K. \& Wilson, M. A. Temporally structured replay of awake hippocampal ensemble activity during rapid eye movement sleep. Neuron (2001) doi:10.1016/S08966273(01)00186-6.

13. Hennevin, E., Hars, B., Maho, C. \& Bloch, V. Processing of learned information in paradoxical sleep: relevance for memory. Behav. Brain Res. (1995) doi:10.1016/01664328(95)00013-J.

14. Cordi, M. J., Diekelmann, S., Born, J. \& Rasch, B. No effect of odor-induced memory reactivation during REM sleep on declarative memory stability. Front. Syst. Neurosci. (2014) doi:10.3389/fnsys.2014.00157.

15. Sterpenich, V. et al. Memory Reactivation during Rapid Eye Movement Sleep Promotes 
Its Generalization and Integration in Cortical Stores. Sleep 37, 1061-1075 (2014).

465

466

467

468

469

470

471

472

473

474

475

476

477

478

479

480

481

482

483

484

485

486

487

488

489

490

491

492

493

494

495

496

497

498

499
16. Rihm, J. S. \& Rasch, B. Replay of conditioned stimuli during late REM and stage N2 sleep influences affective tone rather than emotional memory strength. Neurobiol. Learn. Mem. (2015) doi:10.1016/j.nlm.2015.04.008.

17. Schönauer, M. et al. Decoding material-specific memory reprocessing during sleep in humans. Nat. Commun. (2017) doi:10.1038/ncomms15404.

18. Lee, A. K. \& Wilson, M. A. Memory of sequential experience in the hippocampus during slow wave sleep. Neuron 36, 1183-94 (2002).

19. Eichenlaub, J. B. et al. Replay of Learned Neural Firing Sequences during Rest in Human Motor Cortex. Cell Rep. (2020) doi:10.1016/j.celrep.2020.107581.

20. Nishida, M., Pearsall, J., Buckner, R. L. \& Walker, M. P. REM sleep, prefrontal theta, and the consolidation of human emotional memory. Cereb. Cortex (2009) doi:10.1093/cercor/bhn155.

21. Sederberg, P. B., Kahana, M. J., Howard, M. W., Donner, E. J. \& Madsen, J. R. Theta and Gamma Oscillations during Encoding Predict Subsequent Recall. J. Neurosci. (2003) doi:10.1523/jneurosci.23-34-10809.2003.

22. Fell, J., Klaver, P., Elger, C. E. \& Fernández, G. The interaction of rhinal cortex and hippocampus in human declarative memory formation. Reviews in the Neurosciences (2002) doi:10.1515/REVNEURO.2002.13.4.299.

23. Battaglia, F. P., Benchenane, K., Sirota, A., Pennartz, C. M. A. \& Wiener, S. I. The hippocampus: Hub of brain network communication for memory. Trends in Cognitive Sciences (2011) doi:10.1016/j.tics.2011.05.008.

24. Fell, J. et al. Medial temporal theta/alpha power enhancement precedes successful memory encoding: Evidence based on intracranial EEG. J. Neurosci. (2011) doi:10.1523/JNEUROSCI.3668-10.2011.

25. Vertes, R. P. Hippocampal theta rhythm: A tag for short-term memory. Hippocampus (2005) doi:10.1002/hipo.20118.

26. Poe, G. R., Nitz, D. A., McNaughton, B. L. \& Barnes, C. A. Experience-dependent phasereversal of hippocampal neuron firing during REM sleep. Brain Res. (2000) doi:10.1016/S0006-8993(99)02310-0.

27. Spencer, R. M. C., Sunm, M. \& Ivry, R. B. Sleep-Dependent Consolidation of Contextual Learning. Curr. Biol. (2006) doi:10.1016/j.cub.2006.03.094.

28. Born, J. \& Wilhelm, I. System consolidation of memory during sleep. Psychological Research (2012) doi:10.1007/s00426-011-0335-6.

29. King, J.-R. \& Dehaene, S. Characterizing the dynamics of mental representations: the temporal generalization method. Trends Cogn. Sci. 18, 203-210 (2014). 
30. Koopman, A. C. M. et al. Targeted memory reactivation of a serial reaction time task in SWS, but not REM, preferentially benefits the non-dominant hand. bioRxiv 2020.11.17.381913 (2020) doi:10.1101/2020.11.17.381913.

31. Euston, D. R., Tatsuno, M. \& McNaughton, B. L. Fast-forward playback of recent memory sequences in prefrontal cortex during sleep. Science (80-. ). (2007) doi:10.1126/science.1148979.

32. Murphy, M., Stickgold, R., Parr, M. E., Callahan, C. \& Wamsley, E. J. Recurrence of taskrelated electroencephalographic activity during post-training quiet rest and sleep. Sci. Rep. 8, 1-10 (2018).

33. Diekelmann, S. \& Born, J. The memory function of sleep. Nature Reviews Neuroscience (2010) doi:10.1038/nrn2762.

34. Biel, A. L., Minarik, T. \& Sauseng, P. EEG Cross-Frequency Phase Synchronization as an Index of Memory Matching in Visual Search. Neuroimage (2021) doi:10.1016/j.neuroimage.2021.117971.

35. Magosso, E., Ricci, G. \& Ursino, M. Alpha and theta mechanisms operating in internalexternal attention competition. J. Integr. Neurosci. (2021) doi:10.31083/j.jin.2021.01.422.

36. Hölscher, C., Anwyl, R. \& Rowan, M. J. Stimulation on the positive phase of hippocampal theta rhythm induces long-term potentiation that can be depotentiated by stimulation on the negative phase in area CA1 in vivo. J. Neurosci. (1997) doi:10.1523/jneurosci.1716-06470.1997.

37. Kahana, M. J., Sekuler, R., Caplan, J. B., Kirschen, M. \& Madsen, J. R. Human theta oscillations exhibit task dependence during virtual maze navigation. Nature (1999) doi:10.1038/21645.

38. Schreiner, T. \& Rasch, B. Boosting vocabulary learning by verbal cueing during sleep. Cereb. Cortex (2015) doi:10.1093/cercor/bhu139.

39. Davidson, T. J., Kloosterman, F. \& Wilson, M. A. Hippocampal Replay of Extended Experience. Neuron 63, 497-507 (2009).

40. Diba, K. \& Buzsáki, G. Forward and reverse hippocampal place-cell sequences during ripples. Nat. Neurosci. (2007) doi:10.1038/nn1961.

41. Rothschild, G. The transformation of multi-sensory experiences into memories during sleep. Neurobiol. Learn. Mem. (2019) doi:10.1016/j.nlm.2018.03.019.

42. Schreiner, T., Petzka, M., Staudigl, T. \& Staresina, B. P. Endogenous memory reactivation during sleep in humans is clocked by slow oscillation-spindle complexes. Nat. Commun. (2021) doi:10.1038/s41467-021-23520-2.

43. Mölle, M., Marshall, L., Gais, S. \& Born, J. Grouping of spindle activity during slow oscillations in human non-rapid eye movement sleep. J. Neurosci. (2002) 
doi:10.1523/jneurosci.22-24-10941.2002.

538 44. Antony, J. W., Schönauer, M., Staresina, B. P. \& Cairney, S. A. Sleep Spindles and Memory Reprocessing. Trends in Neurosciences (2019) doi:10.1016/j.tins.2018.09.012.

45. Hasselmo, M. E. Temporally structured replay of neural activity in a model of entorhinal cortex, hippocampus and postsubiculum. Eur. J. Neurosci. 28, 1301-1315 (2008).

542

46. Maquet, P. et al. Experience-dependent changes in cerebral activation during human REM sleep. Nat. Neurosci. 3, 831-836 (2000).

544

47. Peigneux, P. et al. Learned material content and acquisition level modulate cerebral reactivation during posttraining rapid-eye-movements sleep. Neuroimage 20, 125-134 (2003).

48. Cousins, J. N., El-Deredy, W., Parkes, L. M., Hennies, N. \& Lewis, P. A. Cued memory reactivation during slow-wave sleep promotes explicit knowledge of a motor sequence. J. Neurosci. 34, 15870-15876 (2014).

550

49. Oostenveld, R., Fries, P., Maris, E. \& Schoffelen, J. M. FieldTrip: Open source software for advanced analysis of MEG, EEG, and invasive electrophysiological data. Comput. Intell. Neurosci. (2011) doi:10.1155/2011/156869.

553 50. Treder, M. MVPA-Light. (2020). 